\title{
PREFACE: SUPERCRITICAL CARBON DIOXIDE IN THERMAL AND ENERGY SCIENCES
}

Any substance has a critical point which is obtained at certain conditions of pressure and temperature. When a fluid is subjected to a pressure and a temperature higher than its critical point, the fluid is said to be supercritical. In the supercritical region, the thermophysical properties exhibit unique behavior and have intermediate values between those of a liquid and a gas. The supercritical fluids have densities like liquids, viscosities like gases while diffusivities are intermediate in relation to both a liquid and a gas. Supercritical fluids have no surface tension because they are not subject to a vapor-liquid interface, and accordingly no molecules have attraction to the interior of the liquid. The fluid is said to be supercritical as it is heated above its critical temperature and compressed above its critical pressure.

Carbon dioxide $\left(\mathrm{CO}_{2}\right)$ is the most widely used supercritical fluid. This is so as $\mathrm{CO}_{2}$ is cheap, chemically inert, nontoxic, nonflammable, and available at high purities and low costs. In addition, the critical point of $\mathrm{CO}_{2}$ is low, $31^{\circ} \mathrm{C}$ and the critical pressure is 74 bar. These allow the fluid to be used at mild temperature operating conditions without generating any harmful organic residues. It is also sometimes referred to as a green solvent.

Based on the importance of supercritical $\mathrm{CO}_{2}$, a symposium "Advances in Supercritical Carbon Dioxide $\left(\mathrm{SCO}_{2}\right)$ in Thermal and Energy Sciences" (ASTES 2018) was held in Xi'an, China and hosted by Xi'an Jiaotong University, Xi'an, China in July 2018. The major topic areas were: a) thermal and mechanical characteristics of $\mathrm{SCO}_{2}$ in thermal power systems, b) design and analysis of $\mathrm{SCO}_{2}$ heat exchangers for thermal energy systems, c) fluid flow, heat transfer, and energy conversion with $\mathrm{SCO}_{2}$, d) optimization design of $\mathrm{SCO}_{2}$ power and energy systems and components, and e) $\mathrm{SCO}_{2}$ in solar energy, geothermal energy, waste heat.

Some papers were invited for this special issue of Heat Transfer Research (HTR). All papers were reviewed according to the rules and procedures of HTR. We thank all authors for their careful contribution to this special issue and their efforts to maintain a high quality of their papers.

We also thank the editor-in-chief Professor Yong Tao and the managing editor Professor Zhixiong Guo for accepting our proposal of this special issue. The Begell House team is acknowledged for timely and precise handling of this issue.

Guest Editors

Bengt Sunden

Lund University, Lund, Sweden

Gongnan Xie

Northwestern Polytechnical University, Xi'an, China

Yonghui Xie

Xi'an Jiaotong University, Xi'an, China 